\title{
Analysis of the elastic properties of CNTs and their effect in polymer nanocomposites
}

\author{
Manuel Laspalas ${ }^{1, *}$, Agustín Chiminelli ${ }^{1}$, Carlos Saenz ${ }^{1}$, Francisco Serrano ${ }^{1}$ and Clara \\ Valero $^{1}$ \\ ${ }^{1}$ ITAINNOVA (Technological Institute of Aragon),7 María de Luna st, 50018 Zaragoza, Spain
}

\begin{abstract}
A study about of the influence of CNTs elastic properties in polymer nanocomposites is presented. These properties are assumed to be dependent on the CNTs diameter and number of walls, identified as key characteristics of these reinforcements. The analysis is carried out using a micromechanical model based on the mean field homogenization theory for the prediction of the composite elastic properties of a MWCNT - epoxy resin system. A transversally isotropic elastic behavior has been considered for the CNTs based on values/dependencies reported in the literature. Interphase properties between the CNT and epoxy resin has been investigated by means of molecular dynamics simulations.
\end{abstract}

\section{Introduction}

In the past few years, carbon-based nanocomposites has attracted remarkably increasing interest owing to what they offer in terms of improvement of thermal, electrical and mechanical properties. Due to their specific properties, nanoscale size, huge aspect ratio and surface area, the addition of carbon nanotubes (CNTs) can notably modify the polymers responses. Depending on the synthesis procedure used, CNTs are generally produced covering different ranges of diameters and lengths. Moreover, different number of walls can be obtained. These parameters affect the CNTs reinforcement characteristics and, consequently, the resulting nanocomposite properties. In terms of the mechanical response, the influence of the CNTs diameter, length and number of walls in the elastic properties of CNTs has been studied in several works using different techniques. In general, significant differences can be observed in the values/dependencies reported. Then, an analysis of their effects on the mechanical properties of CNT reinforced composites is valuable.

\section{Properties of MWCNT}

Properties of CNTs have been estimated based on numerical methods, like molecular simulation methods, discrete continuum methods, etc. [1-4].

\footnotetext{
* Corresponding author: mlaspalas@itainnova.es
} 
Although most of the references found only report values for the longitudinal modulus, few references are related to the determination of the full set of transversely isotropic properties of SWCNT and MWCNT [1,5-6].

\subsection{Closed expressions for transversely isotropic properties of MWCNTs}

In this work the Shen [1-3] analytical closed expressions are considered, derived by means of an energy approach in the framework of molecular mechanics. These expressions allow determining the sensitivity to the selected key parameters: the CNTs diameter and the number of walls. Although some of these properties are also dependent on the length, the values taken into account in this work are the ones corresponding to high lengths, where the expressions become independent of it.

The graphs in Fig. 1. show a set of five independent elastic constants that fully define a transversely isotropic material analyzing a range of diameters from $0.68 \mathrm{~nm}$ to $10.17 \mathrm{~nm}$ and the number of layers from 1 to the maximum corresponding to each diameter.
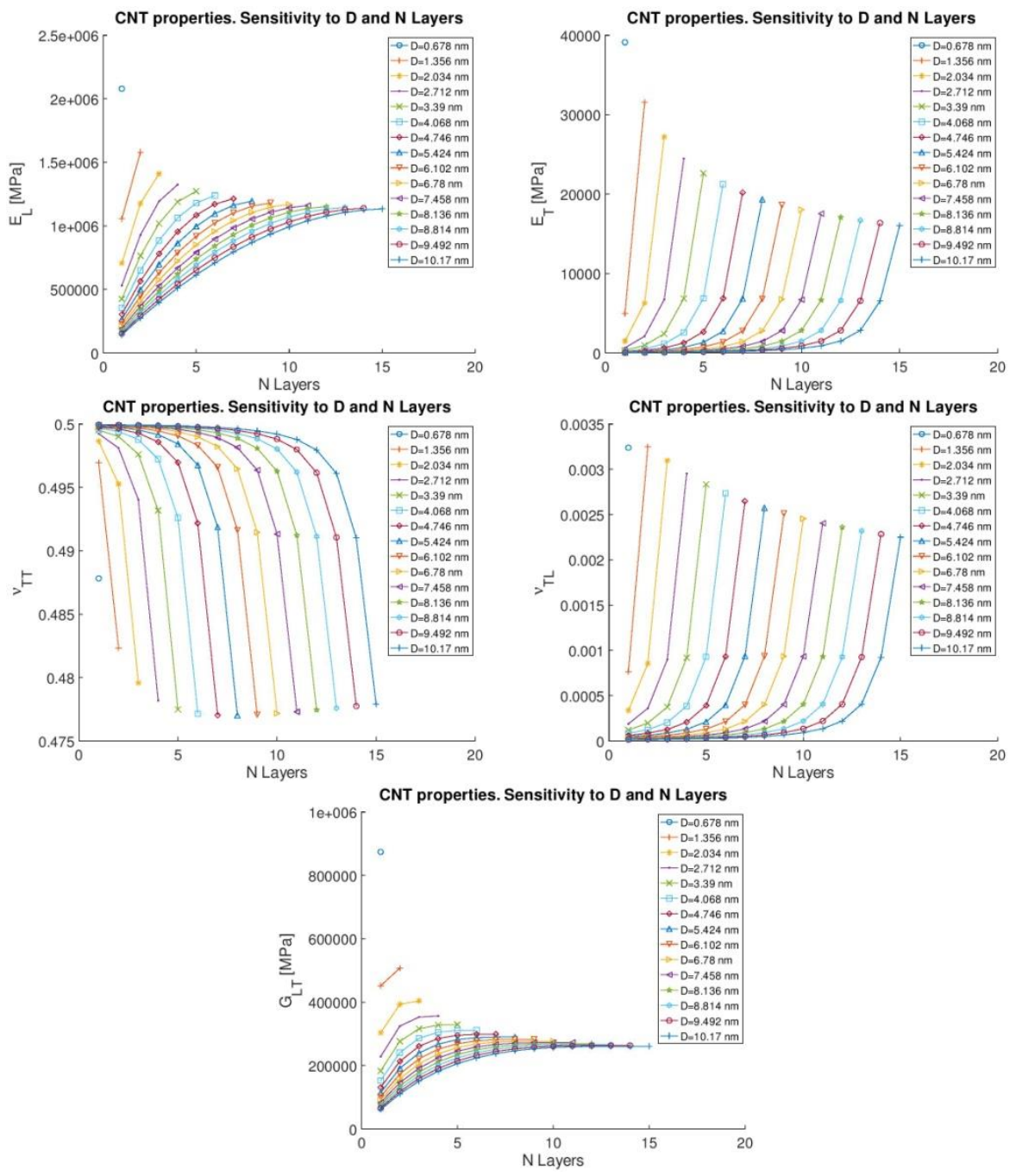

Fig. 1. Transversely elastic properties of CNTs. 
For consistency with next sections, the properties are derived as effective moduli considering the CNT as a solid cylinder of diameter equal to the external layer central atomic positions.

As it can be observed the range of variation of the elastic properties of CNTS is quite significant, however, it is interesting to show these properties in terms of specific properties, that is, dividing by the density (shown if Fig. 2).
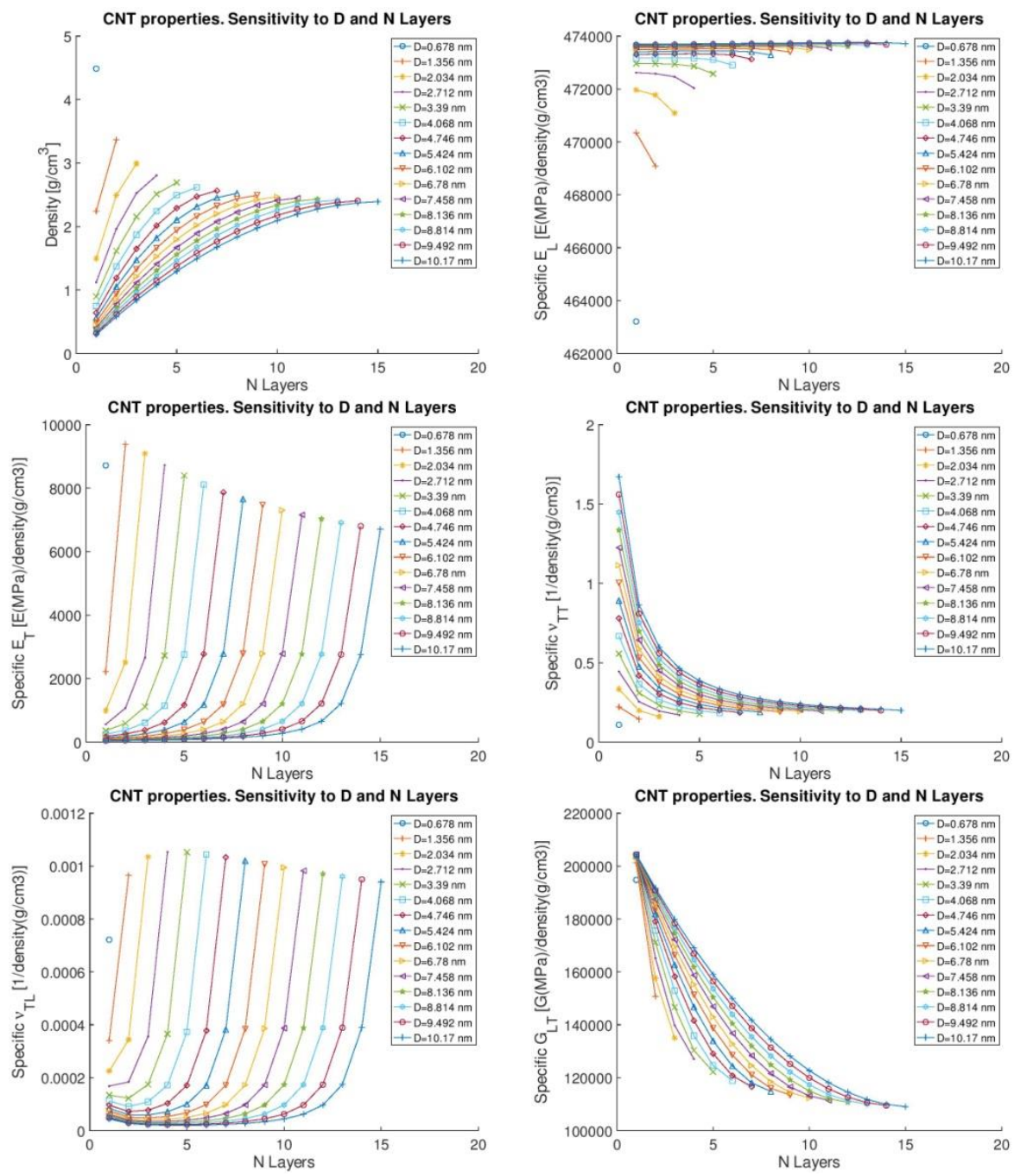

Fig. 2. Specific transversely elastic properties of CNTs.

Table 1 shows a summary of the range of variation, elastic properties and specific elastic properties. It can be observed that, in terms of specific properties, the range of variation is much lower, specially for the axial Young's modulus, that represent approximately a $2 \%$ with regard to the mean of the range limits. The rest of elastic properties show a higher influence.

Table 1. Elastic properties ranges of variation 


\begin{tabular}{|c|c|c|c|c|}
\hline \multirow{2}{*}{ Property } & \multicolumn{2}{|c|}{ Absolute } & \multicolumn{2}{c|}{ Specific } \\
\cline { 2 - 5 } & Min & Max & Min & Max \\
\hline$E_{\mathrm{L}}(\mathrm{MPa})$ & 141734 & 2078986 & 463212 & 473756 \\
\hline $\mathrm{ET}_{\mathrm{T}}(\mathrm{MPa})$ & 11.925 & 39123 & 39.854 & 9383.4 \\
\hline$v_{\mathrm{TT}}$ & 0.47701 & 0.49995 & 0.10869 & 1.6709 \\
\hline$v_{\mathrm{TL}}$ & 0.000013 & 0.0032 & $0.00002 \mathrm{Ta}$ & 0.00105 \\
\hline $\mathrm{GLT}_{\mathrm{LT}}(\mathrm{MPa})$ & 61162 & 874281 & 109031 & 204409 \\
\hline
\end{tabular}

\section{Properties of the interphase between MWCNT and epoxy resin}

\subsection{Outline of the molecular dynamic simulation model}

Nanocomposites are characterized by the formation of interphase regions around the particles with size not negligible with regard to the nanoparticle dimensions, and whose properties are different from the surrounding polymeric matrix. In the case of non functionalized MWCNT the extension of this interphase region and their properties have been investigated through a molecular dynamic simulation (Fig. 3).
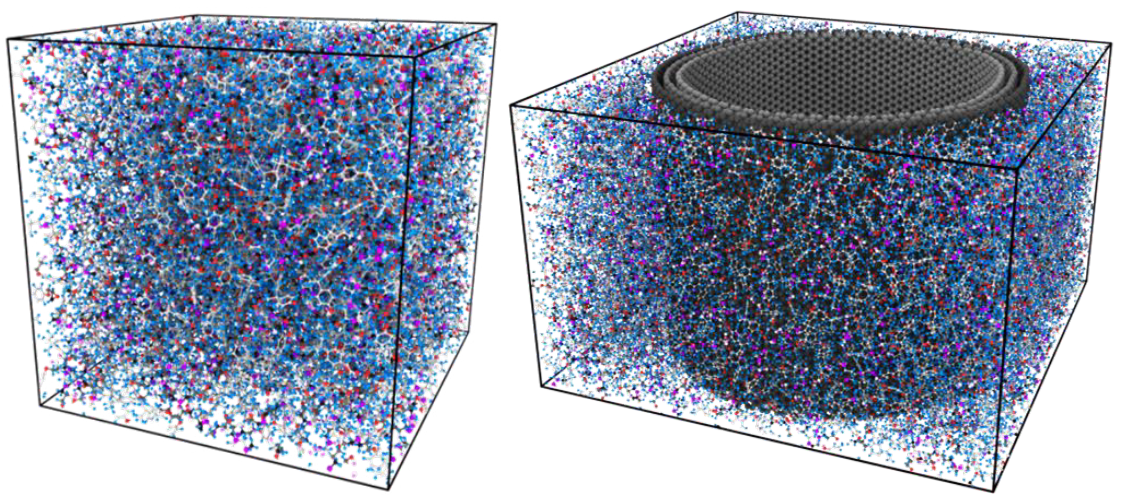

Fig. 3. Equilibrated molecular dynamics model or the pure cured resin (left) and composite (right).

An armchair triple wall CNT (TWCNT) is considered in this analysis. A bond order potential (Tersoff) is used for the $\mathrm{C}-\mathrm{C}$ bond modelling. In addition, to model the interaction between carbon atoms in different layers, the Lennard-Jones 9-6 potential was employed.

The considered epoxy component of the resin was the diglycidyl ether of bisphenol-A (DGEBA) while the hardener is the polyamine dethylene triamine (DETA). The COMPASS force field was used to model interactions between atoms.

After blending the monomers in an isobaric-isotherm ensemble (NPT), the polymer was cross-linked directly in the presence of the carbon reinforcement applying a specifically developed procedure. Finally, time integration in the isobaric-isotherm ensemble (NPT) was repeated until get the plateau in density to get zero stress systems in the cross-linked state.

Then, the full atom system is stretched in different deformation modes to analyze the mechanical response of the nanocomposite (Fig. 4), following an energy minimization procedure considering the static or equilibrium approach. 

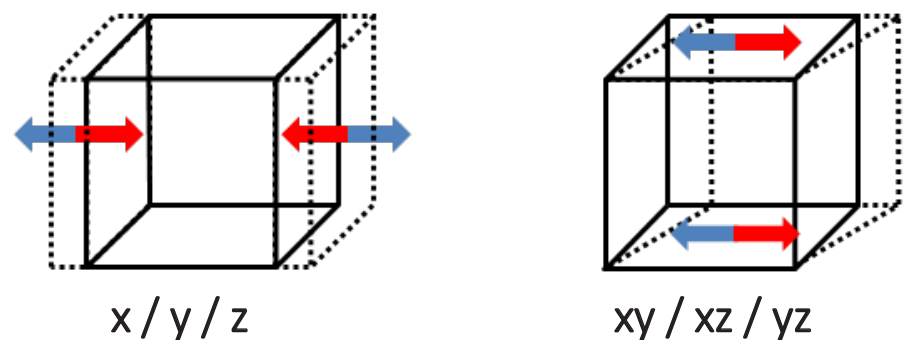

Fig. 4. Longitudinal deformation modes (left), shear deformation modes (right).

\subsection{Results}

From the analysis performed on the pure epoxy resin and in the epoxy resin-TWCNT nanocomposite, the information in Table 2 has been extracted.

Table 2. Elastic properties from MD simulations.

\begin{tabular}{|c|c|c|c|c|}
\hline $\begin{array}{c}\text { Engineering } \\
\text { constant }\end{array}$ & Nanocomposite & TWCNT & Matrix & Interphase \\
\hline $\mathrm{E}_{\mathrm{L}}(\mathrm{MPa})$ & $2.2760 \mathrm{E}+05$ & $4.4042 \mathrm{E}+05$ & \multirow{2}{*}{$4.3503+03$} & $3.7444 \mathrm{E}+03$ \\
\cline { 1 - 3 } $\mathrm{E}_{\mathrm{T}}(\mathrm{MPa})$ & $3.0964 \mathrm{E}+03$ & $8.8761 \mathrm{E}+02$ & & $4.1106 \mathrm{E}+03$ \\
\hline$v_{\mathrm{TT}}$ & $9.1222 \mathrm{E}-01$ & $9.9511 \mathrm{E}-01$ & \multirow{2}{*}{0.31347} & $3.3713 \mathrm{E}-01$ \\
\cline { 1 - 3 }$v_{\mathrm{TL}}$ & $-8.9258 \mathrm{E}-04$ & $-3.0015 \mathrm{E}-04$ & & $3.0701 \mathrm{E}-01$ \\
\hline
\end{tabular}

From radial density distributions from the equilibrated system, the interphase region is quantified to be $1 \mathrm{~nm}$ width. This interphase region, by chosen convention, is considered to include the Van der Waals gap from the external layer of the TWCNT, which is an empty region of around $0.35 \mathrm{~nm}$.

An approach based on considering average strains from dimensions of the cell and the CNT, and average stresses evaluated from the virial stress, has been used to determine the interphase average properties.

It can be seen in Table 2 that resulting interphase properties are slightly lower than the pure matrix, showing that in this system the interphase (including the Van der Waals gap) is not enhancing the mechanical properties. In part, this effect is because having considered the VdW gap belonging to the interphase. It should also mentioned that in this case the CNTs are considered without functionalization, and then there are no chemical bonds /links between the nanotubes and the polymer.

Although this interphase region and properties may be affected by the diameter and number of layers of the CNT, here only one system is included, considered representative of the commercial CNTs being used in further investigations being carried out (Nanocyl NC7000).

\section{Properties of the MWCNT-Epoxy composite}

In order to estimate the properties of the composite with the high aspect ratio typical from commercial CNTs and the very low volume fractions that will represent, the molecular dynamics simulation is not affordable from a computational point of view. Then, a micromechanical homogenization approach, based on mean field theory, is proposed to investigate the effect of the CNT characteristics on the composite properties. 


\subsection{Mean field homogenization theory}

Mean field homogenization approach relies on considering volume averaged fields on all the constituents. The volume averaged strains in a two phases composite is related to their volume fractions:

$$
<\boldsymbol{\varepsilon}_{c}>=V_{\mathrm{i}}<\boldsymbol{\varepsilon}_{\mathrm{i}}>+V_{\mathrm{m}}<\boldsymbol{\varepsilon}_{\mathrm{m}}>=V_{\mathrm{i}}<\boldsymbol{\varepsilon}_{\mathrm{i}}>+\left(1-V_{\mathrm{i}}\right)<\boldsymbol{\varepsilon}_{\mathrm{m}}>
$$

Where $<>$ is volume average, ${ }_{i}$ stands for inclusion, ${ }_{m}$ for the matrix, ${ }_{c}$ for the composite and $V$ is the corresponding volume fraction.

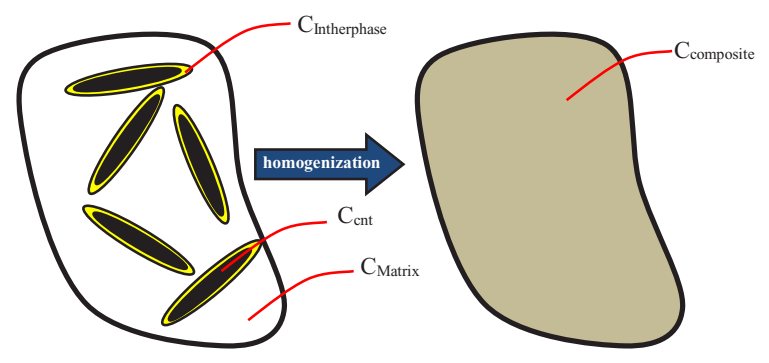

Fig. 5. Homogenization scheme.

Expressing the strains in terms of concentration tensors (2), mean field homogenization determines the composite effective macro stiffness tensor using the expression (3).

$$
\begin{aligned}
& <\boldsymbol{\varepsilon}_{i}>=\boldsymbol{B}_{\mathrm{i}}:<\boldsymbol{\varepsilon}_{\mathrm{m}}> \\
& \boldsymbol{C}_{\mathrm{c}}=\left[V_{\mathrm{i}} \boldsymbol{C}_{\mathrm{i}}: \boldsymbol{B}_{\mathrm{i}}+\left(1-V_{\mathrm{i}}\right) \boldsymbol{C}_{\mathrm{m}}\right]:\left[V_{\mathrm{i}} \boldsymbol{B}_{\mathrm{i}}+\left(1-V_{\mathrm{i}}\right) \boldsymbol{I}\right]^{-1}
\end{aligned}
$$

Then different mean homogenizations approaches differ on the choice of the strain concentration tensor $\left(\boldsymbol{B}_{\mathrm{i}}\right)$, being the most extended the one corresponding to Mori-Tanaka contribution (4).

$$
\boldsymbol{B}_{\mathrm{i}}=\left[\boldsymbol{I}+\boldsymbol{S}: \boldsymbol{C}_{\mathrm{m}}^{-1}:\left(\boldsymbol{C}_{\mathrm{i}}-\boldsymbol{C}_{\mathrm{m}}\right)\right]^{-1}
$$

Being the $\boldsymbol{I}$ the identity tensor, $\boldsymbol{S}$ the Eshelby tensor and $\boldsymbol{C}_{\mathrm{m}}, \boldsymbol{C}_{\mathrm{i}}$ the stiffness tensor of the matrix and the inclusion respectively.

For considering the interphase, the homogenization is performed in two steps. In the first step, the interphase and the CNT is homogenized and the effective inclusion stiffness is determined. In the second step this effective inclusion is homogenized with the rest of the resin.

A relevant issue in composites is the anisotropy produced by the orientation of the reinforcements. CNTs are characterized by high aspect ratio (L/D), but also by their waviness. The degree of orientation will depend on the processing conditions. In this study, a random $3 \mathrm{D}$ distribution is used as basis for comparison. Then, even being the properties of the constituents not isotropic, the resulting material present isotropic symmetry.

\subsection{Sensitivity analysis}

\subsubsection{Number of walls and diameter.}

Based on the model described in section 2 and the properties determined for the matrix and interphase in section 3, a sensitivity analysis for the CNTs diameter and number of walls 
is performed. In this analysis, the aspect ratio (L/D) is fixed to 157.8 , corresponding to the representative value in Nanocyl NC7000 (average diameter of $9.5 \mathrm{~nm}$ and length of $1.5 \mu \mathrm{m}$ ). The weight percentage in this sensitivity analysis is considered to be $1 \mathrm{wt} \%$.
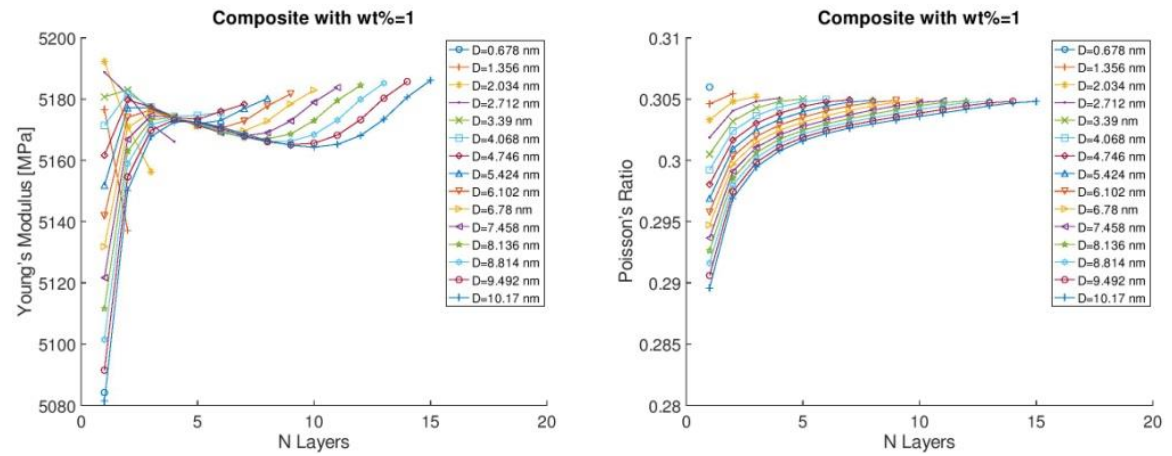

Fig. 6. Sensitivity to diameter and number of walls in Young's modulus (left) and Poisson's ratio (right).

Table 3. Composite Young's modulus.

\begin{tabular}{|c|c|c|c|c|}
\hline Property & Min & Improvement & Max & Improvement \\
\hline Young's modulus $(\mathrm{MPa})$ & 5081.4 & $16.8 \%$ & 5192.3 & $19.4 \%$ \\
\hline
\end{tabular}

The results show that the sensitivity of the elastic constants to the CNTs diameter and number of walls is low, around $2.5 \%$ for the selected $\mathrm{wt} \%$. This is consistent by the previous results shown at CNTs level, since the determinant property of the CNT that shows influence on the nanocomposite is the axial Young's modulus, that in terms of specific values the variation is also very low. Other variables that were much more affected, as the in-plane modulus, or shear transverse modulus, do not play a significant role in the resulting composite properties for the considered random 3D orientation.

\subsubsection{Volume fraction and length.}

In order to evaluate the effect of the volume fraction and the aspect ratio of the CNTs, representative data corresponding to Nanocyl NC7000 commercial MWCNTs is used again, in this case a 7 walls MWCNT is selected, that would fit the known density.

Different weight percentages are analyzed, in the range typically found in literature for reinforcing a polymeric matrix.
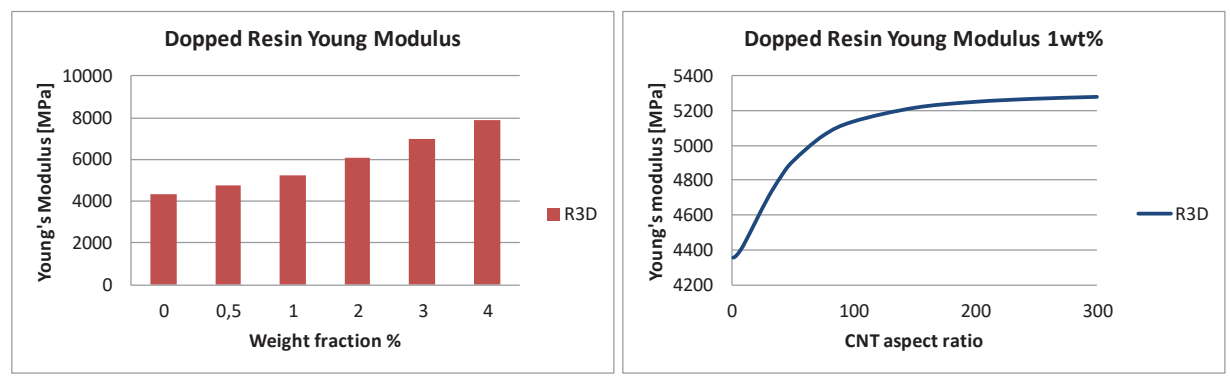

Fig. 7. Sensitivity to weight fraction (left) and aspect ratio (right). 
From the weigh fraction analysis, relevant percentages of improvement for the relative low reinforcement levels considered can be observed. Also the aspect ratio shows a big influence, although for values found on the typical commercial CNTs, the value is close to the plateau region.

\section{Conclusions}

The following conclusions are extracted:

- Although the elastic properties of the MWCNT are highly dependent on the diameter and number of walls, when they are expressed in specific terms, the differences are much lower. Especially for the axial Young's modulus the differences are irrelevant.

- The nanocomposite elastic properties follow the same tendency as the MWCNT. For a given weight fraction of CNTs of $1 \mathrm{wt} \%$, the Young's modulus increases between $16.8 \%$ and $19.4 \%$.

- Relative small weight fractions of CNTs produce a significant enhancement on the elastic properties of the epoxy resin, even in the case the CNTs are not functionalized to produce a stronger interphase region.

- The effect of the interphase properties on the CNT diameter and number of walls has not been analyzed in this study. If a stronger interphase is obtained, for example by functionalizing the CNTs, this effect could represent a significant contribution.

\section{Acknowledges}

This work has been carried out thanks to the funding received from the European Union's Horizon 2020 Research \& Innovation Programme - MODCOMP project under grant agreement No 685844 .

\section{References}

1. L. Shen, J. Li, Physical Review B, 69, 045414, (2004).

2. K.G.S. Dilrukshi et Al, Theoretical and Applied Mechanics Letters 5, 167-172, (2015).

3. J. Doh, J. Lee, Computers and Structures 169, 91-100, (2016).

4. T.H. Nam et Al, Composites: Part A, 76, 289-298 (2015).

5. L. Shen, J. Li, Physical Review B, 81, 119902(E) (2010)

6. L. Shen, J. Li, Physical Review B, 71, 035412, (2005). 